\title{
ON THE EXISTENCE OF CONFORMAL MEASURES
}

\author{
MANFRED DENKER AND MARIUSZ URBAŃSKI
}

\begin{abstract}
A general notion of conformal measure is introduced and some basic properties are studied. Sufficient conditions for the existence of these measures are obtained, using a general construction principle. The geometric properties of conformal measures relate equilibrium states and Hausdorff measures. This is shown for invariant subsets of $S^{1}$ under expanding maps.
\end{abstract}

\section{INTRODUCTION}

Let $(X, \mathscr{F})$ be a measurable space and $T$ be a measurable endomorphism of $X$. In this note, measures $m$ (later called conformal) are studied which have the property that $m \circ T$ is (locally) absolutely continuous with respect to $m$, and the Radon-Nikodym derivative is a continuous function $f$. This type of measures plays an important role in studying the equilibrium theory in dynamics and the fractal nature of attractors and nonwandering sets. There are quite a few contributions using these measures, but there has been no attempt to study these measures in more generality.

The main problem is this: Given a continuous function $f$ on the compact metric space $X$, does there exist a measure $m$, so that $m \circ T$ is (locally) absolutely continuous with Radon-Nikodym derivative $f$ ?

In case of a topologically mixing subshift of finite type (and hence whenever Markov partitions exist) this question has a satisfactory solution by the Schauder-Tychonov theorem (see [1]). If $(X, T)$ is a mixing repeller [16], the existence of conformal measures is easily verified, and this observation avoids constructing Markov partitions to prove, for example, the Bowen-Ruelle formula (first observed by C. Seck). For rational maps on their Julia set $X$, and if the function $f$ denotes a suitable power of the modulus of the derivative of $T$, the problem of constructing conformal measures has been investigated in [18]. In the expanding case it leads to some interesting results about the relation between Gibbs states and Hausdorff measures. In all other cases only little is known. First results, after this paper was written, were obtained in [21] and [22].

The term "conformal measures" is chosen from [12] (see also [13]), where this

Received by the editors September 22, 1989.

1980 Mathematics Subject Classification (1985 Revision). Primary 58F11.

Research supported by the Deutsche Forschungsgemeinschaft, SFB 170, Göttingen. 
type of measure is obtained on limit sets of finitely generated discrete subgroups of conformal mappings acting on hyperbolic space. Again, using these measures, it is possible to study the connection between Hausdorff dimension and the exponent of convergence for Poincaré series.

The first result in $\S 2$ shows that conformal measures for the function $f$ are fixed points of the adjoint of the transfer operator (Perron-Frobenius operator) defined by $f$. It follows from this fact that conformal measures were constructed for some one-dimensional transformations in [6], [7] and [23]. The forthcoming papers [4], [21] and [22] show that there are far reaching applications of conformal measures to the theory of equilibrium states and to the study of Hausdorff dimension and measures. $\S 2$ also gives some basic properties of conformal measures needed in the sequel.

In $\S 3$, a general principle for constructing conformal measures is presented, when $T$ is a continuous, finite-to-one transformation such that there is only a finite number of points, where $T$ is not, locally, a homeomorphism. These points will be called singular. The approach to construct these measures is different from [12], though it has some similarities. It is also different from [6], [7] or [23], where the transfer operator is extended to some larger space, so that it acts on continuous functions and the Schauder-Tychonov theorem applies. It will be shown that $\exp (c-f)$-conformal measures in a weak sense always exist for any continuous function $f$ and some $c \in \mathbf{R}$. These measures turn out to be conformal, if they do not assign positive mass to orbits of singular points. The constant $c$ can be identified with the pressure $P(T, f)$ in case when $P(T, f)>\sup _{x \in X} f(x)$. Theorem 3.13 gives sufficient conditions for this.

One of the applications of the general construction principle and the existence theorem for conformal measures can be found in $\S 4$. Let $T: S^{1} \rightarrow S^{1}$ be a $C^{1+\varepsilon}$-expanding map of the circle. On certain closed, $T$-invariant subsets $X$ (see [20]) and for a Lipschitz continuous function $f$ there exist, by Theorem 3.13 in $\S 3$, an $\exp (P(T, f)-f)$-conformal measure $m_{f}$ and an absolutely continuous equilibrium state $\mu_{f}$ for $f$. Then, using the properties of the conformal measure $m_{f}$, the relation between $\mu_{f}$ and Hausdorff measures $H_{\tilde{h}}$ (see [15] for a definition) on the topological support of $\mu_{f}$ is investigated, where

$$
\tilde{h}(t)=t^{\kappa} \exp \left(\sigma \chi^{-1 / 2} h(-\log t) \sqrt{-\log t}\right) \quad(t>0) .
$$

(Here $\kappa$ denotes the Hausdorff dimension of $\mu_{f}, \chi$ the Lyapunov exponent, $\sigma^{2}$ some asymptotic variance, and $h$ a positive, nondecreasing function (see $\S 4$ for precise definitions).) If $h$ is a lower class function, $\mu_{f}$ is shown to be absolutely continuous with respect to $H_{\tilde{h}}$, and if $h$ is constant, then both measures are orthogonal. In particular, $\mu_{f}$ is singular to the Hausdorff measure corresponding to the function $t^{\kappa}$. This holds whenever $\sigma^{2}>0$. If it is zero, both measures are shown to be equivalent.

This type of problem has been studied in different situations. Fractal prop- 
erties of paths of Brownian motion (and even of more general self-similar processes) have been investigated by several authors (see [5] for further references). Much more related to the present study are investigations of harmonic measures on the boundary of an open topological disc on the Riemann sphere (see [9] or [14] for more details). The latter paper [14] deals with harmonic measures and, more generally, with the images of Gibbs states on conformal mixing repellers and uses, in a dynamical context, the theory of upper and lower class functions from probability and Pesin's theory from dynamics. Although there are some similarities of the present work with that paper, the investigations here lead to some new problems caused by the fact, that, in general, the equilibrium states are no longer Gibbs measures. These difficulties are overcome using conformal measures.

The following notations will be used throughout the paper. Let $X$ be a measurable or metric space. For a subset $A \subset X$, the closure of $A$ is denoted by $\bar{A}$ and the boundary of $A$ by $\partial A$. For $x \in X, r>0$ and $n \geq 1$ let

$$
B_{n}(x, r)=\left\{y \in X: \operatorname{dist}\left(T^{j}(x), T^{j}(y)\right)<r \quad \forall 0 \leq j<n\right\} .
$$

If $n=1, B_{1}(x, r)=B(x, r)$ is the ball with radius $r$ around $x$. Now let $(X, \mathscr{F})$ and $\left(Y, \mathscr{F}^{\prime}\right)$ be measurable spaces and let $T: X \rightarrow Y$ be a measurable map. $T$ will be called a measurable isomorphism, if $T$ is invertible and both $T$ and $T^{-1}$ are measurable. For a measurable function $g$ on $Y$ set $S_{n} g=$ $\sum_{0 \leq k<n} g \circ T^{j}$. Occasionally, $\mu(g)$ will be written for $\int_{Y} g d \mu$, where $\mu$ is a measure on $Y$ and $g \in L_{1}(\mu)$. Finally, $\mathbf{R}_{+}=[0, \infty)$ denotes the set of nonnegative reals and $\mathbf{N}$ the set of nonnegative integers. The cardinality of a set $E$ will be denoted by $\sharp E$. Throughout most of the paper the conformal measures will be probabilities.

\section{Conformal Measures}

Consider a measurable endomorphism $T: X \rightarrow X$ on a measurable space $(X, \mathscr{F})$ and a measurable nonnegative function $f$ on $X$.

Definition 2.1. A measure $m$ on $(X, \mathscr{F})$ is called $f$-conformal, if

$$
m(T A)=\int_{A} f(z) m(d z),
$$

whenever $A \in \mathscr{F}$ is a measurable set, for which $T A$ is measurable and $T: A \rightarrow$ $T A$ is invertible.

In the sequel, a set $A$ as in the definition will be called special. Note that (2.1) implies that $m \circ T$ is absolutely continuous with respect to $m$ on the $\sigma$-algebra $\mathscr{F} \cap A$ for every special set $A$ such that $T: A \rightarrow T A$ is a measurable isomorphism. In this case, (2.1) is equivalent to the fact that the corresponding Radon-Nikodym derivative $d m \circ T / d m=f_{\mid A}$.

Examples. There are several examples of conformal measures appearing in the literature. 
(1) Let $(X, T)$ denote a topologically mixing subshift of finite type and $f$ a Hölder continuous function on $X$. Denote by $L \varphi(x)=\sum_{T(y)=x} \varphi(y) \exp (f(y))$ the Perron-Frobenius operator associated to $f$, which acts on the space of continuous functions $\varphi$ on $X$. By the Schauder-Tychonov theorem, there exists a fixed point of the map $\mathscr{L}(\mu)=\left(L^{*} \mu(1)\right)^{-1} L^{*} \mu$ (see [1, p. 14]). This fixed point is a conformal measure with respect to the function $\exp (P(T, f)-f)$. $(P \cdot, \cdot)$ denotes the pressure function (see [3]).) It is not too difficult to see this, and, if the pressure vanishes, it follows immediately from Proposition 2.2 below.

(2) For any piecewise differentiable map $T$ on an interval, on $S^{1}$ or on $\mathbf{R}^{n}$, Lebesgue measures is conformal with respect to the modulus of the derivative of $T$. In [6] and [7] conformal measures were constructed for piecewise differentiable maps on the unit interval and certain continuous functions, in particular all Lipschitz continuous functions. These measures are shown to be fixed points of the adjoint of the transfer operator and thus, by Proposition 2.2 below, conformal. For $\beta$-transformations the corresponding result has been obtained earlier [23].

(3) Lebesgue measure is conformal with respect to $\left|T^{\prime}\right|$ for any rational map $T$ on the Riemann sphere, but not necessarily for this map restricted to its Julia set. In the latter situation conformal measures were obtained in [18] with respect to the function $\left|T^{\prime}\right|^{\delta}$ for some $\delta \in(0,2]$. In the expanding case, $\delta$ is known to be the Hausdorff dimension of the Julia set [18].

(4) Let $\Gamma$ be a finitely generated discrete subgroup of conformal maps acting on hyperbolic space. In this case, one can construct a measure, which is conformal with respect to the functions $j(g)^{\delta}$ for any map $g \in \Gamma$ acting on the limit set of $\Gamma$ (see [12] and also [13]), where $j$ denotes the conformal distortion. $\delta$ is known to be the exponent of convergence of the Poincare series associated to $\Gamma$.

Let $m$ be an $f$-conformal measure and define $f^{(1)}=f$ and

$$
f^{(n)}=\left(f^{(n-1)} \circ T\right) f \quad \text { for } n \geq 2 .
$$

It follows immediately from (2.1), that for every $n \geq 1, m$ is also $f^{(n)}$. conformal with respect to the transformation $T^{n}$ and

$$
\int_{T A} g d m=\int_{A}(g \circ T) f d m
$$

for any measurable function $g$, which is nonnegative or integrable on $T A$.

Let $T$ be a measurable endomorphism of $(X, \mathscr{F})$, which is finite-to-one (i.e. the numbers of preimages of points is uniformly bounded). For a measurable function $f: X \rightarrow \mathbf{R}_{+}$the Perron-Frobenius operator

$$
L_{f} \varphi(x)=L \varphi(x)=\sum_{T(y)=x} \varphi(y) / f(y)
$$

is well defined for (real-valued) measurable functions $\varphi$ and all $x \in X$ such that $f(y)>0$ for all preimages $y$ of $x$. In this situation one has 
Proposition 2.2. Assume that there exists a finite partition of $X$ into special sets $X_{i}(1 \leq i \leq s)$, such that $T: X_{i} \rightarrow T X_{i}$ is a measurable isomorphism. Let $f: X \rightarrow \mathbf{R}_{+}$be a measurable function and $m$ be a probability measure on $(X, \mathscr{F})$. Then $m$ is $f$-conformal if and only if $L_{f}$ acts on $L^{1}(m)$ and $L^{*} m=m$.

Proof. Let $M$ be $f$-conformal. By (2.1), for $1 \leq i \leq s, m\left(T A_{i}\right)=0$ where $A_{i}=\left\{x \in X_{i}: f(x)=0\right\}$. Whence $L \varphi$ is a.e. well defined. Moreover, if $\varphi$ is an integrable function, (2.2) implies

$$
\int_{T X_{i}} \frac{\varphi}{f} \circ\left(T_{\mid X_{i}}\right)^{-1} d m=\int_{X_{i}} \varphi d m \quad(1 \leq i \leq s) .
$$

Summing over $i$ yields

$$
\int_{X} L_{f} \varphi d m=\int_{X} \varphi d m
$$

Conversely, assume that $L_{f}$ acts on $L^{1}(m)$ and that $m$ is a fixed point of $L^{*}$. Let $A$ be a special set. Then, by (2.3),

$$
\begin{aligned}
\int_{A} f d m & =\int_{A} f d L^{*} m=\int_{X} L\left(1_{A} f\right) d m \\
& =\int_{X} \sum_{T(y)=x} 1_{A}(y) m(d x)=m(T A) .
\end{aligned}
$$

Thus $m$ is $f$-conformal.

Definition 2.3. Let $X$ be a compact metric space and $T: X \rightarrow X$ be a continuous map, which is finite-to-one. Denote by $X_{0}$ the set of all points $x \in X$, such that for all sufficiently small $\varepsilon>0$ the set $T B(x, \varepsilon)$ is not open. A point $x \in X$ is called critical (for $T$ ), if there does not exist an open neighborhood of $x$, which is a special set, and it is called singular, if it is critical or if it belongs to $X_{0}$.

Denote by $\operatorname{Crit}(T)$ (resp. $\operatorname{Sing}(T)=X_{0} \cup \operatorname{Crit}(X)$ ) the set of critical (resp. singular) points for $G$. If $T$ has only a finite number of singular points, it follows that for every nonsingular point $x \in X$ there exists an open neighbourhood $U$ of $x$, such that $T_{\mid U}$ is a homeomorphism onto the open set $T U$. Moreover, if $m$ is a Borel measure on the Borel $\sigma$-field $\mathscr{F}$, then there exists a finite measurable partition of $X(\bmod m)$ into special sets on which $T$ acts as a measurable isomorphism. In fact, this can be proved, for example, applying Zorn's lemma to the system

$$
\left\{\left(A_{1}, \ldots, A_{s}\right): m\left(A_{i} \cap A_{j}\right)=0(i \neq j) ; T_{\mid A_{i}} \text { is a measurable isomorphism }\right\},
$$

where $s$ is sufficiently large, and where the ordering is given by inclusion $\bmod m$.

For the remaining part of this section, let $X$ be a compact metric space and $T: X \rightarrow X$ be a continuous map, which is finite-to-one, and which has at most a finite number of singular points. 
Lemma 2.4. Let $m$ be a measure on $(X, \mathscr{F})$ and let $f \in L^{1}(m)$. Assume that (2.1) holds for every special set $C \in \mathscr{F}$ with $C \cap \operatorname{Sing}(T)=\varnothing$ and $m(\partial C)=$ $m(\partial T C)=0$. Then for any special set $A \in \mathscr{F}$ satisfying $A \cap \operatorname{Sing}(T)=\varnothing$, (2.1) continues to hold.

Proof. Let $\varepsilon>0$. For each $x \in A$ there exists an open set $C(x)$ such that $T_{\mid C(x)}$ is a homeomorphism, $m(\partial C(x))=m(\partial T C(x))=0, C(x) \cap \operatorname{Sing}(T)=$ $\varnothing$, and such that

$$
\int_{\bigcup C(x) \backslash A} f d m<\varepsilon
$$

The existence of sets with these properties follows from well-known measure theoretic arguments, since $T$ is open at $x \in A$. Choose a countable family $\left\{C_{k}\right\}$ from $\{C(x)\}$ which covers $A$ and define recursively $A_{1}=C_{1}$ and $A_{n}=$ $C_{n} \backslash \bigcup_{k<n} C_{k}$.

By definition and by the assumption in the lemma, each set $A_{k}$ satisfies (2.1) and hence

$$
\begin{aligned}
m(T A) & =m\left(\bigcup_{k=1}^{\infty} T\left(A \cap A_{k}\right)\right) \leq \sum_{k=1}^{\infty} m\left(T A_{k}\right) \\
& =\sum_{k=1}^{\infty} \int_{A_{k}} f d m=\int_{A} f d m+\sum_{k=1}^{\infty} \int_{A_{k} \backslash A} f d m \\
& \leq \int_{A} f d m+\varepsilon .
\end{aligned}
$$

If $\varepsilon \rightarrow 0$, it follows that $m(T B) \leq \int_{B} f d m$ for any special set $B$ not containing any singular point.

Using this fact, the lower bound for $m(T A)$ is obtained from the following estimate, if $\varepsilon \rightarrow 0$ :

$$
\begin{aligned}
m(T A) & =m\left(\bigcup_{k=1}^{\infty} T\left(A \cap A_{k}\right)\right)=\sum_{k=1}^{\infty} m\left(T\left(A \cap A_{k}\right)\right) \\
& =\sum_{k=1}^{\infty}\left[m\left(T A_{k}\right)-m\left(T\left(A_{k} \backslash A\right)\right)\right] \\
& \geq \sum_{k=1}^{\infty}\left[\int_{A_{k}} f d m-\int_{A_{k} \backslash A} f d m\right] \\
& =\int_{\bigcup_{k \geq 1} A_{k}} f d m-\int_{\bigcup_{k \geq 1} A_{k} \backslash A} f d m \geq \int_{A} f d m-\varepsilon .
\end{aligned}
$$

This proves the lemma.

Theorem 2.5. Let $T: X \rightarrow X$ be a continuous, finite-to-one map of the compact metric space $X$ having at most a finite number of singular points, and let $f_{n}$ be measurable functions. For each $n \in \mathbf{N}$, let $m_{n}$ be an $f_{n}$-conformal normalized 
Borel measure, such that

$$
K:=\sup _{n \geq 1}\left\|\frac{f_{n}}{f_{n} \circ T}\right\|_{L^{\infty}\left(m_{n}\right)}<\infty, \text { or } \sup _{n \geq 1}\left\|f_{n}\right\|_{L^{1}\left(m_{n}\right)}<\infty,
$$

and such that $\left(m_{n}: n \geq 1\right)$ converges weakly to some measure $m$. Moreover, suppose that $m$ satisfies the following condition:

$$
m(\{x\})=0 \Rightarrow m(\{T(x)\})=0 \quad(\forall x \in \operatorname{Sing}(T)) .
$$

Then $m$ is $f$-conformal for some $f \in L^{1}(m)$.

Proof. It suffices to prove the theorem under the assumption

$$
K:=\sup _{n \geq 1}\left\|\frac{f_{n}}{f_{n} \circ T}\right\|_{L^{\infty}\left(m_{n}\right)}<\infty .
$$

The other case is proved similarly, but in a simpler way.

Let $\left\{X_{1}, \ldots, X_{s}\right\}$ be a finite measurable partition $\left(\bmod m_{n}\right)$ into special sets. (This can be obtained simultaneously for all $n \in \mathbf{N}$ by the remark preceding Lemma 2.4.) Define measures $\mu_{n}$ by $\mu_{n}(A)=\int_{A} f_{n} d m_{n}(m \geq 1)$. Then

$$
\begin{aligned}
\mu_{n}(X) & =\int_{X} f_{n} d m_{n}=\sum_{i=1}^{s} \int_{X_{i}} f_{n} d m_{n} \\
& =\sum_{i=1}^{s} m_{n}\left(T X_{i}\right) \leq s .
\end{aligned}
$$

Therefore there is no loss of generality assuming that the measures $\mu_{n}$ converge weakly to some measure $\mu$ with $\mu(X) \leq s$.

Next, using (2.2),

$$
\begin{aligned}
\left\|f_{n}\right\|_{2}^{2} & =\sum_{i=1}^{s} \int_{X_{i}} f_{n}^{2} d m_{n}=\sum_{i=1}^{s} \int_{T X_{i}} f_{n} \circ\left(T_{\mid X_{i}}\right)^{-1} d m_{n} \\
& \leq K \sum_{i=1}^{s} \int_{T X_{i}} f_{n} d m_{n} \leq s K \int_{X} f_{n} d m_{n} \\
& =s K \sum_{i=1}^{s} \int_{X_{i}} f_{n} d m_{n}=s K \sum_{i=1}^{s} m_{n}\left(T X_{i}\right) \\
& \leq s^{2} K<\infty .
\end{aligned}
$$

For any set $U \in \mathscr{F}$ satisfying $\mu(\partial U)=m(\partial U)=0$ it follows that

$$
\begin{aligned}
\mu(U) & =\lim _{n \rightarrow \infty} \mu_{n}(U)=\lim _{n \rightarrow \infty} \int_{U} f_{n} d m_{n} \\
& \leq \lim _{n \rightarrow \infty}\left\|f_{n}\right\|_{2}\left(m_{n}(U)\right)^{1 / 2} \leq\left(s^{2} K m(U)\right)^{1 / 2} .
\end{aligned}
$$

Clearly, this suffices to show that $\mu$ is absolutely continuous with respect to $m$, and so, by the Radon-Nikodym theorem, there exist a function $\tilde{f} \in L^{1}(m)$ satisfying $\mu(A)=\int_{A} \tilde{f} d m$ for $A \in \mathscr{F}$. 
Define $f$ by setting $f(x)=\tilde{f}(x)$ if $x \in X$ is nonsingular. This defines $f$ a.e., if $m$ assigns measure zero to $\operatorname{Sing}(T)$. If $x \in X$ is singular and $m(\{x\})>0$, then set

$$
f(x)=m(\{T(x)\}) / m(\{x\}) .
$$

It is left to show that $m$ is $f$-conformal. First note that for a set $C \in \mathscr{F}$ with $C \cap \operatorname{Sing}(T)=\varnothing$ and $\mu(\partial C)=m(\partial T C)=0$, one has

$$
\begin{aligned}
m(T C) & =\lim _{n \rightarrow \infty} m_{n}(T C)=\lim _{n \rightarrow \infty} \int_{C} f_{n} d m_{n} \\
& =\lim _{n \rightarrow \infty} \mu_{n}(C)=\mu(C)=\int_{C} \tilde{f} d m=\int_{C} f d m .
\end{aligned}
$$

By (2.4) and (2.6), it suffices to check the condition (2.1) for special sets $A$ not containing any singular point. By (2.7) the assumptions in Lemma 2.4 are satisfied, whence the theorem.

Corollary 2.6. Assume that the measure $m$ in Theorem 2.5 assigns measure zero to $\operatorname{Sing}(T)$. If $f_{n}$ converges uniformly to some function $f$, then $m$ is $f$-conformal.

Proof. This follows from (2.5).

Corollary 2.7. If in Corollary 2.6, $f_{n}=s_{n} h$ for some continuous function $h$ and some $s_{n} \in \mathbf{R}_{+}$, and if $s=\lim _{n \rightarrow \infty} s_{n}$ exists, then $m$ is sh-conformal.

It is possible to obtain a similar theorem about weak convergence to some conformal measure using a version of the proof of Proposition 3.11 below. We state it without proof. (In fact, its proof is contained in the proof of Theorem 3.13.)

Theorem 2.8. Let $T: X \rightarrow X$ be a continuous, finite-to-one map of the compact metric space $X$ having at most a finite number of singular points. Assume that $X_{0} \cap \operatorname{Crit}(T)=\varnothing$ and that $T$ has no critical periodic point. Let $f_{n}, f$ be continuous functions so that $\left(f_{n}: n \geq 1\right)$ converges uniformly to $f$. If $\inf _{x \in X} f(x)>1$ and if the $f_{n}$-conformal measures $m_{n}$ converge weakly to $m$, then $m$ is $f$ conformal.

\section{CONSTRUCTIONS OF CONFORMAL MEASURES}

The main purpose of this section is to provide a general scheme for constructing conformal measures. Although the basic idea goes back to [12], the construction turns out to be different, and is, in fact, more closely related to the proof of the variational principle for the pressure in [11]. Besides this, several sufficient conditions for the existence of conformal measures are presented.

Throughout this section let $X$ be a compact metric space, equipped with its Borel $\sigma$-field $\mathscr{F}$, and let $T: X \rightarrow X$ be a continuous map, such that the set $\operatorname{Sing}(T)$ of singular points is finite. Moreover, let $f: X \rightarrow \mathbf{R}$ be a continuous 
function and fix (except otherwise stated) a sequence $\left(E_{n}: n \geq 1\right)$ of finite subsets of $X$ satisfying

$$
T^{-1} E_{n} \subset E_{n+1} \quad \forall n \in \mathbf{N}
$$

The construction principle for conformal measures will make use of the following simple analytical fact. For a sequence $\left(a_{n}: n \geq 1\right)$ of reals, the number $c=\limsup _{n \rightarrow \infty} a_{n} / n$ will be called the transition parameter of $\left(a_{n}: n \geq 1\right)$. It is uniquely determined by the fact that $\sum_{n \in \mathbf{N}} \exp \left(a_{n}-n s\right)$ converges for $s>c$ and diverges for $s<c$. For $s=c$ the sum may converge or diverge. By a simple argument (see e.g. [12] for a similar consideration) one obtains

Lemma 3.1. There exists a sequence $\left(b_{n}: n \geq 1\right)$ of positive reals such that

$$
\sum_{n=1}^{\infty} b_{n} \exp \left(a_{n}-n s\right) \begin{cases}<\infty, & s>c \\ =\infty, & s \leq c\end{cases}
$$

and $\lim _{n \rightarrow \infty} b_{n} / b_{n+1}=1$.

Proof. If $\sum \exp \left(a_{n}-n c\right)<\infty$, choose a sequence $n_{k} \in \mathbf{N}(k \geq 1)$ satisfying

$$
\lim _{k \rightarrow \infty} n_{k} n_{k+1}^{-1}=0 \quad \text { and } \quad \varepsilon_{k}:=a_{n_{k}} n_{k}^{-1}-c \rightarrow 0 \text {. }
$$

Setting

$$
b_{n}=\exp \left(n\left(\frac{n_{k}-n}{n_{k}-n_{k-1}} \varepsilon_{k-1}+\frac{n-n_{k-1}}{n_{k}-n_{k-1}} \varepsilon_{k}\right)\right) \text { for } n_{k-1} \leq n<n_{k},
$$

it is easy to check the claim.

Construction Principle. Given the function $f$ and the finite sets $E_{n}(n \geq 1)$, the general construction is done as follows: Let $a_{n}=\log \sum_{x \in E_{n}} \exp S_{n} f(x)$, where $S_{n} f=\sum_{0 \leq k<n} f \circ T^{k}$. Denote by $c$ the transition parameter of this sequence. Choose a sequence $\left(b_{n}: n \geq 1\right)$ of positive reals as in Lemma 3.1 for the sequence $\left(a_{n}: n \geq 1\right)$. For $s>c$ define

$$
M_{s}=\sum_{n=1}^{\infty} b_{n} \exp \left(a_{n}-n s\right)
$$

and the normalized measure

$$
m_{s}=\frac{1}{M_{s}} \sum_{n=1}^{\infty} \sum_{x \in E_{n}} b_{n} \exp \left(S_{n} f(x)-n s\right) \delta_{x}
$$

where $\delta_{x}$ denotes the unit mass at the point $x \in X$. 
Let $A \in \mathscr{F}$ be a special set. Using (3.1) and (3.3) it follows that

$$
\begin{aligned}
m_{s}(T A)= & \frac{1}{M_{s}} \sum_{n=1}^{\infty} \sum_{x \in E_{n} \cap T A} b_{n} \exp \left(S_{n} f(x)-n s\right) \\
= & \frac{1}{M_{s}} \sum_{n=1}^{\infty} \sum_{x \in A \cap T^{-1} E_{n}} b_{n} \exp \left(S_{n} f(T(x))-n s\right) \\
= & \frac{1}{M_{s}} \sum_{n=1}^{\infty} \sum_{x \in A \cap E_{n+1}} b_{n} \exp \left[S_{n+1} f(x)-(n+1) s\right] \exp (s-f(x)) \\
& -\frac{1}{M_{s}} \sum_{n=1}^{\infty} \sum_{x \in A \cap\left(E_{n+1} \backslash T^{-1} E_{n}\right)} b_{n} \exp \left(S_{n} f(T(x))-n s\right) .
\end{aligned}
$$

Define

$$
\begin{aligned}
\Delta_{A}(s)=\mid \frac{1}{M_{s}} \sum_{n=1}^{\infty} \sum_{x \in A \cap E_{n+1}} b_{n} \exp \left[S_{n+1} f(x)-(n+1) s\right] & \exp (s-f(x)) \\
& -\int_{A} \exp (c-f) d m_{s} \mid
\end{aligned}
$$

and observe that

$$
\begin{aligned}
& \Delta_{A}(s)= \frac{1}{M_{s}} \mid \sum_{n=1}^{\infty} \sum_{x \in A \cap E_{n+1}} \exp \left[S_{n+1} f(x)-(n+1) s\right] \\
& \cdot \exp (-f(x))\left[b_{n} e^{s}-b_{n+1} e^{c}\right]-b_{1} \sum_{x \in A \cap E_{1}} e^{c-s} \mid \\
& \leq \frac{1}{M_{s}} \sum_{n=1}^{\infty} \sum_{x \in A \cap E_{n+1}}\left|\frac{b_{n}}{b_{n+1}}-e^{c-s}\right| b_{n+1} \exp (s-f(x)) \\
& \leq \frac{1}{M_{s}} \sum_{n=1}^{\infty} \sum_{x \in E_{n+1}}\left|\frac{b_{n}}{b_{n+1}}-e^{c-s}\right| b_{n+1} \exp (s-f(x)) \\
& \cdot \exp \left[S_{n+1} f(x)-(n+1) s\right]+\frac{1}{M_{s}} b_{1} \exp (c-s) \sharp\left(A \cap E_{1}\right) \\
& \cdot \exp \left[S_{n+1} f(x)-(n+1) s\right]+\frac{1}{M_{s}} b_{1} \exp (c-s) \sharp E_{1} .
\end{aligned}
$$

By Lemma 3.1, $\lim _{n \rightarrow \infty} b_{n+1} / b_{n}=1$ and $\lim _{s \downarrow c} M_{s}=\infty$. Therefore

$$
\lim _{s \downarrow c} \Delta_{A}(s)=0
$$

uniformly for all special sets $A \in \mathscr{F}$.

Definition 3.2. Any weak accumulation point, when $s \downarrow c$, of the measures $\left(m_{s}: s>c\right)$ defined in the construction principle will be called a limit measure (associated to the function $f$ and the sequence $\left(E_{n}: n \geq 1\right)$ ). 
In order to find conformal measures among the limit measures, it is necessary to examine (3.4) in greater detail. For a subset $D \in \mathscr{F}$ consider the condition

$$
\lim _{s \downarrow c} \frac{1}{M_{s}} \sum_{n=1}^{\infty} \sum_{x \in D \cap\left(E_{n+1} \backslash T^{-1} E_{n}\right)} b_{n} \exp \left[S_{n} f(T(x))-n s\right]=0 .
$$

Lemmas 3.3-3.5 collect a few useful facts about the behaviour of a limit measure at singular points and on special sets, which do not contain a singular point.

Lemma 3.3. Let $m$ be a limit measure. Assume that every special set $D \in \mathscr{F}$ with $m(\partial D)=m(\partial T D)=0$, and $\bar{D} \cap \operatorname{Sing}(T)=\varnothing$ satisfies condition (3.6). Then for any special set $A \in \mathscr{F}$ with $A \cap \operatorname{Sing}(T)=\varnothing$,

$$
m(T A)=\int_{A} \exp (c-f) d m .
$$

Proof. Let $D \in \mathscr{F}$ be a special set such that $\bar{D} \cap \operatorname{Sing}(T)=\varnothing$ and $m(\partial D)=$ $m(\partial T D)=0$. It follows immediately from (3.4)-(3.6) that

$$
m(T D)=\int_{D} \exp (c-f) d m .
$$

Now, let $A$ be as in the lemma, and let $C \subset A$ be compact. A slight modification of the proof of Lemma 2.4 shows that (3.7) continues to hold for $C$. Since every Borel measuie is regular, and since $T$ is continuous, (3.7) also holds for $A$ by approximation with compact sets contained in $A$.

Lemma 3.4. Assume that condition (3.6) is satisfied for $D=X$. Then for $x \in$ $\operatorname{Sing}(T) \backslash \operatorname{Crit}(T)$

$$
m(\{T(x)\}) \geq \exp (c-f(x)) m(\{x\}) .
$$

Proof. Let $\left(C_{n}: n \geq 1\right)$ be a sequence of compact neighbourhoods of $x \in$ $\operatorname{Sing}(T) \backslash \operatorname{Crit}(T)$, decreasing to $x$, so that each $C_{n}$ is special and satisfies $m\left(\partial C_{n}\right)=0$. From (3.4), (3.5), and the assumption, one obtains

$$
\lim _{s \in J}\left|m_{s}\left(T C_{n}\right)-\int_{C_{n}} \exp (c-f) d m_{s}\right|=0 \quad \forall n \geq 1,
$$

where $J$ denotes the subsequence along which $m_{s}$ converges to $m$. Since $T C_{n}$ is compact, this clearly implies

$$
\begin{aligned}
m\left(T C_{n}\right) & \geq \limsup _{s \in J} m_{s}\left(T C_{n}\right) \\
& =\lim _{s \in J} \int_{C_{n}} \exp (c-f) d m_{s} \\
& =\int_{C_{n}} \exp (c-f) d m .
\end{aligned}
$$

Letting $n \rightarrow \infty$, the lemma follows.

Lemma 3.5. Assume that condition (3.6) holds for all sets $D \in \mathscr{F}$ satisfying $\bar{D} \cap X_{0}=\varnothing$. Then for every limit measure $m$ and every point $x \in \operatorname{Crit}(T) \backslash X_{0}$, 
one has $m(\{x\})=0 \Leftrightarrow m(\{T(x)\})=0$. More precisely,

$$
m(\{T(x)\}) \leq \exp (c-f(x)) m(\{x\}) \leq q(x) m(\{T(x)\})
$$

where $q=q(x)$ denotes the number of preimages of $T$ restricted to a sufficiently small neighbourhood of $x$.

Proof. Since $x \notin X_{0}$, there exist open neighbourhoods $U$ of $x$ of arbitrarily small diameter, containing no other singular point and satisfying $m(\partial U)=$ $m(\partial T U)=0$. Observe that there exists a decomposition of $U$ into special sets $U_{1}, \ldots, U_{q}$. Then, using (3.4)-(3.6),

$$
\begin{aligned}
\lim _{s \in J} \sum_{i=1}^{q} m_{s}\left(T U_{i}\right) & =\lim _{s \in J} \sum_{i=1}^{q} \int_{U_{i}} \exp (s-f) d m_{s} \\
& =\lim _{s \in J} \int_{U} \exp (s-f) d m_{s}=\int_{U} \exp (c-f) d m,
\end{aligned}
$$

where $J$ denotes the subsequence determined by the convergence to $m$. Since $m_{s}(T U) \leq \sum m_{s}\left(T U_{i}\right) \leq q m_{s}(T U)$ for every $s>c$, it follows that

$$
m(T U) \leq \int_{U} \exp (c-f) d m \leq q m(T U),
$$

and the lemma follows letting $U \downarrow x$.

Remark 3.6. It seems important to note the following consequence of the foregoing three lemmas. If $m$ is a limit measure and if (3.6) is satisfied for $D=X$, then $m$ satisfies $(2.1)$ for all special sets not intersecting $\operatorname{Sing}(T)$, and

$$
\begin{aligned}
m(\{T(x)\}) & \geq \exp (c-f(x)) m(\{x\}) \quad(x \in \operatorname{Sing}(T) \backslash \operatorname{Crit}(T)), \\
m(\{T(x)\}) & \leq \exp (c-f(x)) m(\{x\}) \\
& \leq q(x) m(\{T(x)\}) \quad\left(x \in \operatorname{Crit}(T) \backslash X_{0}\right) .
\end{aligned}
$$

This implies that $m$ is $\exp (c-f)$-conformal, if $m(T \operatorname{Sing}(T))=0$ and the measure on orbits of points in $\operatorname{Crit}(T) \cap X_{0}$ is zero.

Definition 3.7. A Borel probability measure $m$ is called weakly $\exp (c-f)$ conformal, if

$$
m(T A)=\int_{A} \exp (c-f) d m
$$

for all special sets $A$ not intersecting $\operatorname{Sing}(T)$.

The preceding lemmas imply the following results.

Proposition 3.8. There exists a weakly $\exp (p-f)$-conformal Borel probability measure $m$ for some $p \in \mathbf{R}$. Moreover, $m$ satisfies

$$
\begin{aligned}
m(\{T(x)\}) & \geq \exp (p-f(x)) m(\{x\}) \quad(x \in \operatorname{Sing}(T) \backslash \operatorname{Crit}(T)), \\
m(\{T(x)\}) & \leq \exp (p-f(x)) m(\{x\}) \\
& \leq q(x) m(\{T(x)\}) \quad\left(x \in \operatorname{Crit}(T) \backslash X_{0}\right) .
\end{aligned}
$$


Proof. Since $T$ is continuous and $X$ is compact, there exists some point $z \in$ $\bigcap_{n=0}^{\infty} T^{n} X$. Setting $E_{n}=T^{-n}\{z\}$, and defining $p$ by the transition parameter associated to $\left(E_{n}: n \geq 1\right)$ and $f$, the proposition follows immediately from Lemmas 3.3, 3.4 and 3.5.

Theorem 3.9. Let $T$ be an open map and let $m$ be a limit measure (associated to the function $f$ and the sequence $\left(E_{n}: n \geq 1\right)$ ) assigning measure zero to any critical periodic point. Moreover, assume that condition (3.6) is satisfied for $D=X$. Then there exists an $\exp (c-f)$-conformal measure $\tilde{m}$. Moreover, if $m(\operatorname{Crit}(T))=0$, then $m=\tilde{m}$.

Remark. If $T$ is a rational map on the Riemann sphere, there exists an $\exp (p-f)$-conformal measure on the Julia set of $T$ for some $p \in \mathbf{R}$. This follows from the previous theorem and proposition, and the fact that no critical periodic point of a rational map can be contained in the Julia set.

Proof. By Lemmas 3.3, 3.4 and 3.5 the measure $m$ has the properties stated in (3.8). Since $T$ is open, $m$ is $\exp (c-f)$-conformal, if $m(\operatorname{Crit}(T))=0$. (See Remark 3.6.)

Denote by $Y$ the union of all forward and backward orbits of critical points. If $m(Y)<1$, it is easy to see that, restricting $m$ to $X \backslash Y$, gives an $\exp (c-f)$ conformal measure.

In the remaining case, it is not too difficult to define a conformal measure on the orbit of a critical point. For simplicity in notation, let us assume that $x_{0}$ is a critical point and that its orbit $O\left(x_{0}\right)$ contains no other critical point. Again, inducing if necessary, w.l.o.g. $m\left(O\left(x_{0}\right)\right)=1$. By Lemma 3.5 there exists $\alpha>0$ such that $m\left(\left\{T\left(x_{0}\right)\right\}\right)=\alpha m\left(\left\{x_{0}\right\}\right)$. Define a new measure $\tilde{m}$ by setting

$$
\tilde{m}(\{x\})=\left\{\begin{array}{l}
\beta m(\{x\}) \quad \text { if } T^{n}(x)=x_{0} \text { for some } n \geq 0, \\
\beta \alpha^{-1} \exp \left(c-f\left(x_{0}\right)\right) m(\{x\}) \text { if not }
\end{array}\right.
$$

where

$$
\begin{aligned}
\beta^{-1}= & m\left(\left\{x: T^{n}(x)=x_{0} \text { for some } n \geq 0\right\}\right) \\
& +m\left(\left\{x \in O\left(x_{0}\right): T^{n}(x) \neq x_{0} \text { for all } n \geq 0\right\}\right) \alpha^{-1} \exp \left(c-f\left(x_{0}\right)\right) .
\end{aligned}
$$

It is easy to see that $\tilde{m}$ is $\exp (c-f)$-conformal.

Example 3.10. The previous theorem is not true, if the map $T$ is not open. There is a very simple example for this. For every integer $n \geq 1$ let $x_{n}=$ $\exp (2 \pi i / n)$ and $X=\left\{x_{n}: n \geq 1\right\}$. Define the transformation $T: X \rightarrow X$ setting

$$
T\left(x_{n}\right)=\left\{\begin{array}{l}
x_{n-1} \text { for } n \geq 2 \\
x_{n} \text { for } n=1
\end{array}\right.
$$

Clearly, $X$ is compact and $T$ is continuous, surjective and even invertible for all $x_{n}(n \geq 2)$. For $f$ being the constant function 0 there is no $\exp c$-conformal measure for any $c \in \mathbf{R}$. Assume that $m$ is $e^{c}$-conformal. Since $m\left(\left\{x_{j}\right\}\right)>0$ 
for some $j \geq 1, m\left(\left\{x_{1}\right\}\right)=m\left(\left\{T^{j-1}\left(x_{j}\right)\right\}\right)=\exp ((j-1) c) m\left(\left\{x_{j}\right\}\right)>0$. Hence $m\left(\left\{x_{1}\right\}\right)=m\left(\left\{T\left(x_{1}\right)\right\}\right)=e^{c} m\left(\left\{x_{1}\right\}\right)$ implies that $c=0$. But then $m\left(\left\{x_{n}\right\}\right)=m\left(\left\{x_{1}\right\}\right)$ for all $n \geq 1$, which is impossible.

Proposition 3.8 implies that conformal measures exist whenever a limit measure is not concentrated on orbits of singular points. In particular this is true, if the limit measure is atom-free. The following proposition ensures this property, even in the case when $X_{0} \neq \varnothing$.

Proposition 3.11. Let condition (3.6) be satisfied for $D=X$. In addition, suppose that $c>\sup _{x \in X} f(x), \quad X_{0} \cap \operatorname{Crit}(T)=\varnothing$, and that no periodic point is critical. Then every limit measure $m$ is an atom-free $\exp (c-f)$-conformal measure.

Proof. Suppose that $m(\{x\})>0$ for some $x \in X$. Since $X_{0} \cap \operatorname{Crit}(T)=$ $\varnothing$, Lemmas 3.3-3.5 imply that $m\left(\left\{T^{n}(x)\right\}\right)>0$ for all $n \geq 0$. Since no periodic point is critical and since $c>\sup _{x \in X} f(x)$, these lemmas also show that $\lim _{n \rightarrow \infty} m\left(\left\{T^{n}(x)\right\}\right)=\infty$, a contradiction. Thus any limit measure is atom-free, and the proposition follows from Lemma 3.3.

Remark. Conditions of the form $c>\sup _{x \in X} f(x)$ appear frequently in the literature. It is, for example, satisfied if

$$
\limsup _{n \rightarrow \infty} \frac{1}{n} \log \sharp E_{n}>\sup _{x \in X} f(x)-\inf _{x \in X} f(x) .
$$

In the remaining part of this section the transition parameter $c$ will be determined, when the sets $E_{n}$ are separated (see [3]). Sufficient conditions will be given to identify the transition parameter with the pressure $P(T, f)$ of $f$. The following notation will be used.

Let $\varepsilon>0$ and let $F_{n}(\varepsilon)(n \geq 1)$ be a sequence of maximal $(n, \varepsilon)$-separated sets. Define

$$
P(\varepsilon)=\limsup _{n \rightarrow \infty} \frac{1}{n} \log \sum_{x \in F_{n}(\varepsilon)} \exp \left(S_{n} f(x)\right) .
$$

Note that $P(\varepsilon)$ is the transition parameter associated to $f$ and the sequence $E_{n}=F_{n}(\varepsilon)(n \geq 1)$ if $T^{-1} F_{n}(\varepsilon) \subset F_{n+1}(\varepsilon)$ (this cannot always be achieved). Then the pressure of $f$ is defined by $P=P(T, f)=\lim _{\varepsilon \rightarrow 0} P(\varepsilon)$. Recall that $P(T, f)$ does not depend on the particular choice of the maximal separated sets, that $P(T, f)<\infty$, if the topological entropy is finite, and that $P(T, f)=P(\delta)$ for any expansive constant $\delta>0$, if $T$ is expansive.

The existence of $\exp (P-f)$-conformal measures will be proved in the following theorems.

Theorem 3.12. There exists a weakly $\exp (P(T, f)-f)$-conformal measure $m$ for any expansive map $T$. If, in addition, $T$ is an open map then $m$ is $\exp (P(T, f)-f)$-conformal.

Proof. Let $\delta>0$ be an expansive constant. Then, by expansiveness, every two preimages of a point in $X$ are at a distance at least $\delta$. Thus there exists a 
sequence $\left(E_{n}: n \geq 1\right)$ of maximal $(n, \delta)$-separated sets $E_{n}$ satisfying $T^{-1} E_{n} \subset$ $E_{n+1}(n \geq 1)$, and hence, by the foregoing remarks, it suffices to show the condition (3.6) for sets $D$ as in Lemma 3.3.

Let $D \in \mathscr{F}$ satisfy $\bar{D} \cap \operatorname{Sing}(T)=\varnothing$. Note that there exists a constant $\gamma>0$ such that

$$
T B(x, \delta) \supset B(T(x), \gamma) \quad \forall x \in D
$$

By expansiveness,

$$
\lim _{n \rightarrow \infty}\left(\sup _{x \in X} B_{n}(T(x), \delta)\right)=0 .
$$

Hence there exists some $n_{0} \geq 1$ such that for all $n \geq n_{0}$ and $x \in X$

$$
\operatorname{diam} B_{n}(x, \delta)<\gamma \text {. }
$$

In order to prove (3.6) it suffices to show

$$
D \cap\left(E_{n+1} \backslash T^{-1} E_{n}\right)=\varnothing \quad \forall n \geq n_{0} .
$$

Assume that $x \in D \cap\left(E_{n+1} \backslash E_{n}\right)$ for some $n \geq n_{0}$. Since $E_{n}$ is maximal separated, there exists a point $y \in E_{n}$ with $T(x) \in B_{n}(y, \delta)$, equivalently $y \in B_{n}(T(x), \delta)$. By (3.11) and (3.12) there is $z \in B(x, \delta)$ with $T(z)=y$. Consequently $x, z \in B_{n+1}(x, \delta) \cap E_{n+1}$, contradicting the separation property of $E_{n+1}$. This proves (3.13) and the first part of the theorem. The additional statement follows, e.g., from Remark 3.6.

Theorem 3.13. Let $G$ be of finite topological entropy and such that $X_{0} \cap \operatorname{Crit}(T)$ $=\varnothing$. Moreover, assume that $G$ has no critical periodic point and that for all sufficiently small $\varepsilon>0$ there exists a sequence $\left(F_{n}(\varepsilon): n \geq 1\right)$ of maximal $(n, \varepsilon)$-separated sets satisfying (3.1) and

$$
\begin{gathered}
\max \left(0 ; \sup _{\varepsilon>0} \limsup _{n \rightarrow \infty} \frac{1}{n} \log \sharp\left(F_{n+1}(\varepsilon) \backslash T^{-1} F_{n}(\varepsilon)\right)\right) \\
<P(T, f)-\sup _{x \in X} f(x) .
\end{gathered}
$$

Then there exists an atom-free $\exp (P(T, f)-f)$-conformal measure on $(X, \mathscr{F})$. Proof. Let $\varepsilon>0$ sufficiently small so that $P(\varepsilon)>d:=\sup _{x \in X} f(x)$, and that the $F_{n}(\varepsilon)$ satisfy the equation (3.14), where the right-hand side is replaced by $P(\varepsilon)-d-\eta$ for some $\eta>0$. The main step in the proof is to show the existence of an $\exp (P(\varepsilon)-f)$-conformal measure $m_{\varepsilon}$.

Since $P(\varepsilon)$ is the transition parameter associated to $f$ and the sequence $\left(F_{n}(\varepsilon): n \geq 1\right)$, in view of Proposition 3.11 the existence of $m_{\varepsilon}$ is guaranteed, once condition (3.6) is verified for $D=X$. By (3.14) and the definition of $b_{n}$, there is an $n_{0} \geq 1$ such that

$$
b_{n} \leq \exp \left(\frac{n}{2} \eta\right) \quad\left(\forall n \geq n_{0}\right)
$$

and

$$
\sharp\left(F_{n+1}(\varepsilon) \backslash T^{-1} F_{n}(\varepsilon)\right)<\exp [n(P(\varepsilon)-d-\eta)] \quad\left(\forall n \geq n_{0}\right) .
$$


It follows for $n \geq n_{0}$ and $s>P(\varepsilon)$ that

$$
\begin{aligned}
& \sum_{n=n_{0}}^{\infty} \sum_{x \in F_{n+1}(\varepsilon) \backslash T^{-1} F_{n}(\varepsilon)} b_{n} \exp \left[S_{n} f(T(x))-n s\right] \\
& \quad \leq \sum_{n=n_{0}}^{\infty} \exp \left[-\frac{n}{2} \eta+n(P(\varepsilon)-s)+n\left(S_{n} f(T(x))-d\right)\right] \\
& \quad \leq \sum_{n=n_{0}}^{\infty} \exp \left[-\frac{n}{2} \eta\right]<\infty .
\end{aligned}
$$

Since $\lim _{s \downarrow P(\varepsilon)} M_{s}=\infty,(3.6)$ follows.

Finally, consider a weakly convergent subsequence $m_{n}(n \geq 1)$ of the family $\left(m_{\varepsilon}: \varepsilon>0\right)$, when $\varepsilon \downarrow 0$. Denote this limit by $m$. Then for every special set $A$ satisfying $m(\partial A)=m(\partial T A)=0$,

$$
m(T A)=\int_{A} \exp (P-f) d m .
$$

By Lemma 2.4, (3.15) holds for any special set $A$ not intersecting $\operatorname{Sing}(T)$. Hence, it is left to show that $m$ has no atoms.

Let $x \in \operatorname{Sing}(T) \backslash \operatorname{Crit}(T)=X_{0}$. Choose decreasing closed neighbourhoods $C_{k}$ of $x$, which are special and satisfy $m\left(\partial C_{k}\right)=0$. Then

$$
\begin{aligned}
m\left(T C_{k}\right) & \geq \limsup _{n \rightarrow \infty} m_{n}\left(T C_{k}\right) \\
& =\lim _{n \rightarrow \infty} \int_{C_{k}} \exp (P-f) d m_{n} \\
& =\int_{C_{k}} \exp (P-f) d m,
\end{aligned}
$$

and the statement of Lemma 3.4 holds in the present situation also. Similarly, repeating the argument in the proof of Lemma 3.5 it follows that the statement of that lemma also holds for a point $x \in \operatorname{Crit}(T) \backslash X_{0}=\operatorname{Crit}(T)$. By this and (3.15), it follows as in the proof of Proposition 3.11 that $m$ cannot have any atom, and this finishes the proof in view of Corollary 2.7.

Remark 3.14. If $T$ is expansive, the existence of one sequence $\left(F_{n}(\varepsilon): n \geq 1\right)$ for some fixed expansive constant $\varepsilon>0$ suffices in Theorem 3.13. Also note that in this case the above proof simplifies considerably.

\section{EXPANDING MAPS ON $S^{1}$}

The aim of this section is to compare equilibrium states for continuous functions $f$ and certain Hausdorff measures. For this one would like to express the equilibrium measure of balls $B_{n}(x, \delta)$ in terms of partial sums of some associated function (cf. (4.12)). Except for very special cases (subshifts of finite type, for example) this is not possible. However, it can be done for $\exp (P(T, f)-f)$ conformal measures and the equilibrium state for $f$ turns out to be absolutely continuous with respect to this conformal measure. 
Throughout this section, $T$ denotes an expanding map of the circle $S^{1}$ of class $C^{1+\varepsilon}$ and $X \subset S^{1}$ denotes an invariant (i.e., $\left.T X \subset X\right)$, closed subset. To begin with, let us make some assumptions and conventions concerning the notation. Denote by $T_{X}$ the restriction of $T$ to $X$ and by $\mathscr{R}$ the family of all connected components of $S^{1} \backslash X$. Define (see [20])

$$
\tau(X)=\{(a, b) \in \mathscr{R}: X \cap T(a, b) \neq \varnothing\} .
$$

It is always assumed that $\tau(X)$ is a fintie set. Changing, if necessary, the Riemannian metric on $S^{1}$ it is also assumed that

$$
\lambda:=\inf _{x \in S^{1}}\left|T^{\prime}(x)\right|>1 .
$$

Finally, $\delta>0$ denotes an expansive constant, which is smaller than the diameters of sets from $\tau(X)$ and so small that on every arc in $S^{1}$ of diameter less than $\delta$ all inverse branches of $T$ are defined.

Lemma 4.1. There exists a sequence $\left(E_{n}: n \geq 1\right)$ of maximal $(n, \delta)$-separated sets $E_{n} \subset X$ satisfying condition (3.1) and $\sharp\left(E_{n+1} \backslash T_{X}^{-1} E_{n}\right) \leq k$ for some $k$ not depending on $\delta$ or $n$.

Proof. The sets $E_{n}$ will be defined recursively. Let $E_{1}$ be any maximal $(1, \delta)$ separated set. If $E_{n-1}$ is chosen, extend $T_{X}^{-1} E_{n-1}$ to a maximal $(n, \delta)$ separated set $E_{n}$. This is possible, since $\delta$ is an expansive constant, hence $T_{X}^{-1} E_{n-1}$ is already an $(n, \delta)$-separated set.

The lemma will be proved by showing that there exists an injective map $\sigma$ from the set $E_{n+1} \backslash T_{X}^{-1} E_{n}$ into the set $G$ of endpoints of arcs from $\tau(X)$.

Let $z \in E_{n+1} \mid T_{X}^{-1} E_{n}$. Since $E_{n}$ is a maximal separated set, $T(z) \in B_{n}\left(z^{\prime}, \delta\right)$ for some $z^{\prime} \in E_{n}$. Denote by $g: B(T(z), \delta) \rightarrow S^{1}$ the branch of $T^{-1}$ determined by the condition $g(T(z))=z$. Then $g\left(z^{\prime}\right) \notin E_{n+1}$, since $\left|z-g\left(z^{\prime}\right)\right|<\delta$ (by the expanding property (4.2)), $z \in E_{n+1}$ and $z \neq g\left(z^{\prime}\right)$. Consequently, $g\left(z^{\prime}\right) \notin T^{-1} E_{n}$ and hence there exists an interval $(a, b) \in \tau(X)$ with $g\left(z^{\prime}\right) \in$ $(a, b)$. Therefore the arc $B_{n+1}\left(g\left(z^{\prime}\right), \delta\right)$ contains exactly one of the endpoints $a$ or $b$. This endpoint will be denoted by $\sigma(z)$.

It is left to show that $\sigma$ is injective. Assume that $\sigma\left(z_{1}\right)=\sigma\left(z_{2}\right):=c$, where $z_{1}, z_{2} \in E_{n+1} \backslash T_{X}^{-1} E_{n}$. By the above construction of $\sigma$ there exist $\bar{z}_{1}, \bar{z}_{2} \in$ $T^{-1} E_{n}$ such that $\bar{z}_{i} \in B_{n+1}\left(z_{i}, \delta\right)$ and $c$ separates the $\operatorname{arcs}\left(z_{i}, \bar{z}_{i}\right)(i=1,2)$. Moreover, $c$ does not separate the arc $\left(z_{1}, z_{2}\right)$ and hence either

$$
z_{1} \in\left(z_{2}, \bar{z}_{2}\right) \subset B_{n+1}\left(z_{2}, \delta\right) \text { or } z_{2} \in\left(z_{1}, \bar{z}_{1}\right) \subset B_{n+1}\left(z_{1}, \delta\right) .
$$

In either case, $z_{1} \in B_{n+1}\left(z_{2}, \delta\right)$, and so $z_{1}=z_{2}$, since $E_{n+1}$ is maximal separated.

Theorem 4.2. Let the continuous function $f: X \rightarrow \mathbf{R}$ satisfy

$$
P(T, f)>\sup _{x \in X} f(x) .
$$


Then there exists an atom-free $\exp (P(T, f)-f)$-conformal measure $m_{f}$ on $(X, \mathscr{F})$.

Proof. The theorem follows from Theorem 3.13 (in fact Corollary 3.14) and Lemma 4.1. Since there are no critical points, it suffices to show that there are only finitely many points in $X_{0}$, where $X_{0}$ is defined after Definition 2.3.

The proof of Proposition 1 in [20] shows that the set $G$ of endpoints of intervals from $\tau(X)$ can be decomposed into pairs $\left(a_{i}, b_{i}\right),(1 \leq i \leq k)$ such that $X \subset \bigcup_{1 \leq i \leq k}\left[a_{i}, b_{i}\right]$ and $\left(T_{X}\right)_{\|\left[a_{i}, b_{i}\right] \cap X}$ has the Darboux property $\bmod X$, that is: every interval $I \subset\left[a_{i}, b_{i}\right] \cap X$ is mapped by $T_{X}$ onto an interval of $X$. Therefore, every singular point $z$ is either isolated or has an open neighbourhood of the form $[z, a)$ resp. $(a, z]$. In the first case, there are two intervals from the family $\mathscr{R}$ adjacent to $z$, and at least one of them contains preimages of points in $X$. So, at least one of the intervals belongs to $\tau(X)$, and hence the number of these singular points is finite, since $\tau(X)$ is. In the second case, there is an interval from the family $\mathscr{R}$ adjacent to $z$, which must contain a preimage of some point from $X$. Again, the number of these singular points is finite.

Theorem 4.3. Let $f: X \rightarrow \mathbf{R}$ be a Lipschitz continuous function satisfying $P(T, f)-\sup _{x \in X} f(x)>0$, and let $m_{f}$ be an atom-free, $\exp (P(T, f)-f)$ conformal measure on $(X, \mathscr{F})$. Then there exists an ergodic, $T_{X}$-invariant probability measure $\mu_{f}$ on $(X, \mathscr{F})$, which is absolutely continuous with respect to $m_{f}$. Moreover, $\mu_{f}$ is an equilibrium state for $f$, and there exists an integer $k \geq 1$, such that for any function $g$ of bounded variation the process $\left(g \circ T^{k n}: n \geq 1\right)$ is a functional of an absolutely regular process with exponentially fast decreasing mixing coefficients (in the sense of [8], see also [6] and [7]).

Proof. By Proposition 2.2, $m_{f}$ is a fixed point of the Perron-Frobenius operator. Thus the results in [6] (see also [7]) can be applied directly to obtain the theorem.

Remark 4.4. (1) After this paper was written, G. Keller and the authors made the statement of Theorem 4.3 more precise, using Theorem 3.13. Theorem 1 in [4] shows that every equilibrium state is absolutely continuous with respect to some $\exp (P(T, f)-f)$-conformal measure. Hence the existence of $\mu_{f}$ in the previous theorem follows already from expansiveness, and, moreover, every equilibrium state has the property given in Theorem 4.3 for some conformal measure. Therefore, the following results apply to all equilibrium states for $f$.

(2) The preceding theorem generalizes and strengthens the result in [7] in the sense that it provides a larger class of functions, for which the existence of fixed points of the Perron-Frobenius operator can be achieved. It is clear from the present discussion, how the above arguments can be applied in other situations of expansive maps, e.g. subshifts.

(3) The last statement in the preceding theorem has an important conse- 
quence. It implies that the process $\left(g \circ T^{k n}: n \geq 1\right)$ satisfies the central limit theorem and the a.s. invariance principle if

$$
\begin{aligned}
\sigma^{2}= & \sigma^{2}(g)=\int_{X}\left(g-\mu_{f}(g)\right)^{2} d \mu_{f} \\
& +2 \sum_{n=1}^{\infty}\left(g-\mu_{f}(g)\right)\left(g \circ T_{X}^{k n}-\mu_{f}(g)\right) d \mu_{f}
\end{aligned}
$$

is strictly positive. The latter theorem means that one can redefine the process ( $g \circ T_{X}^{k n}: n \geq 1$ ) on some probability space on which there is defined a standard Brownian motion $(B(t): t \geq 0)$ such that for some $\beta>0$

$$
\sum_{0 \leq j \leq t}\left[g \circ T_{X}^{k j}-\mu_{f}(g)\right]-B\left(\sigma^{2} t\right) \ll t^{1 / 2-\beta} \quad \mu_{f} \text { a.e. }
$$

(4) Let $h:[1, \infty) \rightarrow \mathbf{R}$ be a positive nondecreasing function. $h$ is said to belong to the lower class if

$$
\int_{1}^{\infty} \frac{h(t)}{t} \exp \left(-\frac{1}{2} h(t)^{2}\right)<\infty
$$

and to the upper class if

$$
\int_{1}^{\infty} \frac{h(t)}{t} \exp \left(-\frac{1}{2} h(t)^{2}\right)=\infty .
$$

From the third remark and well known results on Brownian motion it follows that (for a function $g$ as in (3))

$$
\mu_{f}\left(\left\{x \in X: \sum_{j=0}^{n-1}\left[g\left(T_{X}^{k j}(x)\right)-\mu_{f}(g)\right]>\sigma h(n) \sqrt{n}\right.\right.
$$

$$
\begin{gathered}
\text { for infinitely many } n \in \mathbf{N}\}) \\
= \begin{cases}0 & \text { if } h \text { belongs to the lower class, } \\
1 & \text { if } h \text { belongs to the upper class, }\end{cases}
\end{gathered}
$$

where $\sigma^{2}$ is defined by (4.3) and strictly positive.

Let $K$ be the topological support of $\mu_{f}$. Since $\mu_{f}$ has no atoms, no isolated point belongs to $K$. Hence $B_{X}(x, \tau):=B(x, r) \cap X$ has a positive diameter for $x \in K$, and so, for an open interval $U$ intersecting $K$, consider integers $n(U)$ satisfying the following two conditions (4.7) and (4.8):

$$
T_{X}^{k n(U)}(U \cap X) \supset B_{X}(x, \delta)
$$

for some $x \in K$ and

$$
T^{k n(U)}: U \rightarrow T^{k n(U)} U
$$

is injective. Note that integers with these properties always exist.

Finally, let $d(U)$ denote the diameter of the set $U \cap X$, where $U$ is an open interval. 
Lemma 4.5. There exists a constant $\alpha>0$ such that for every interval $U \subset S^{1}$

$$
m_{f}(U) \leq(\operatorname{diam} U)^{\alpha} .
$$

Proof. Since $m_{f}$ is positive on open sets of $K$, no matter how small $\delta$ is chosen,

$$
A:=A(\delta)=\inf _{x \in K} m_{f}(B(x, \delta))>0 .
$$

By the distortion theorem (see, for example, Lemma 1 in [17]), there exists a constant $C_{1}>0$ such that for $x \in K$ and $U$ containing $x$

$$
C_{1}^{-1}\left|\left(T^{k n(U)}\right)^{\prime}(x)\right|^{-1} \leq d(U) \leq C_{1}\left|\left(T^{k n(U)}\right)^{\prime}(x)\right|^{-1} .
$$

Since $T^{k n(U)}$ is injective on $U$ and since $f$ is Lipschitz continuous, there exists a constant $C_{2}$ such that

$$
\left|S_{k n(U)} f(x)-S_{k n(U)} f(y)\right| \leq C_{2}
$$

for all $x \in K$, all $U$ containing $x$, and $y \in U$. Therefore, by conformality and (4.10), there exists a constant $C_{3}$ such that for $x \in K$ and $U$ containing $x$

$$
\begin{aligned}
& C_{3}^{-1} \exp \left(S_{k n(U)}(f-P(T, f))(x)\right) \\
& \quad \leq m_{f}(U) \leq C_{3} \exp \left(S_{k n(U)}(f-P(T, f))(x)\right) .
\end{aligned}
$$

Since $P(T, f)>\sup _{x \in X} f(x)$, there exists an $\alpha>0$ satisfying

$$
\inf _{x \in X}(P(T, f)-f(x))>\alpha \sup _{x \in X} \log \left|T^{\prime}(x)\right| .
$$

From this, (4.11), and (4.12), one easily deduces the lemma. Note that the full strength of the estimates (4.11) and (4.12) is not needed here, but they will be used later.

Lemma 4.6. For all $\varepsilon>0$ and $n \in \mathbf{N}$, the expansive constant $\delta$ can be chosen so small that

$$
\mu_{f}(\{x \in K: \exists U \text { with } n(U)=n \text { and } x \in U\}) \geq 1-\varepsilon .
$$

Proof: Denote by $G$ the set of endpoints of intervals from $\tau(X)$ and choose $1<\eta^{-1}<\lambda^{k}$. For $n \geq 1$ define

$$
G_{n}=\left\{x \in K: \operatorname{dist}\left(T^{k j}(x), G\right) \geq \eta^{n-j}(\forall 0 \leq j \leq n)\right\} .
$$

By Lemma 4.5,

$$
m_{f}\left(G_{n}\right) \geq 1-\sharp G \sum_{j=0}^{n}\left(\delta \eta^{n-j}\right)^{\alpha} \geq 1-\frac{\delta^{\alpha}}{1-\eta^{\alpha}} \sharp G .
$$

By this estimate and since $\mu_{f} \ll m_{f}, \delta$ may be chosen so small that $\mu_{f}\left(G_{n}\right) \geq$ $1-\varepsilon$. For $x \in G_{n}$ let $U=g B\left(T^{k n}(x), \delta\right)$, where $g$ denotes the branch of 
$T^{-k n}$ determined by $g\left(T^{k n}(x)\right)=x$. Then $n$ may serve as some $n(U)$ as in (4.7) and (4.8), and $x \in U$. It foliows that

$$
G_{n} \subset\{x \in K: \exists U \text { with } n(U)=n \text { and } x \in U\},
$$

and the lemma is proved.

Lemma 4.7. For every constant $h$ the expansive constant $\delta$ can be chosen so small, such that for every function $g$ of bounded variation

$$
\begin{aligned}
& \mu_{f}\left(\left\{x \in X: \sum_{j=0}^{n-1}\left[g\left(T_{X}^{k j}(x)\right)-\mu_{f}(g)\right]>\sigma h \sqrt{n}\right.\right. \text { for infinitely many } \\
& \qquad n \in \mathbf{N} \text { satisfying } n=n(U) \text { for some } U \text { with } x \in U\})=1,
\end{aligned}
$$

provided $\sigma^{2}$ defined in (4.3) is larger than zero.

Proof. Let $0<2 \varepsilon<(2 \pi)^{-1 / 2} \int_{h}^{\infty} \exp \left(-u^{2} / 2\right) d u$ and choose $\delta$ as in Lemma 4.6. Define

$$
Z_{n}^{\prime}=\{x \in K: \exists U \text { with } n(U)=n \text { and } x \in U\} .
$$

By Lemma 4.6 and the central limit theorem (see Remark 4.4.3)), the set

$$
Z_{n}=Z_{n}^{\prime} \cap\left\{x \in X: \frac{1}{\sqrt{\sigma^{2} n}} \sum_{j=0}^{n-1}\left(g\left(T^{k j}(x)\right)-\mu_{f}(g)\right)>h\right\}
$$

has measure $>\varepsilon$ for all sufficiently large $n$. The set $Z=\bigcap_{n \in \mathbf{N}} \bigcup_{l>n} Z_{l}$ is invariant under $T$ and has positive measure, whence by ergodicity, $\mu_{f}(Z)=1$. Now, $x \in Z$ belongs to infinitely many $Z_{n}$, consequently for such $n$

$$
\sum_{j=0}^{n-1}\left(g\left(T^{k j}(x)\right)-\mu_{f}(g)\right) \geq h \sigma \sqrt{n}
$$

and $n(U)=n$ for some $U$ containing $x$. This proves the lemma.

Some more notations are used in the sequel. Denote the Lyapunov characteristic exponent by $\chi=\chi_{f}=\int_{X} \log \left|\left(T^{k}\right)^{\prime}\right| d \mu_{f}$ and the Hausdorff dimensin of $\mu_{f}$ by $\kappa=H D\left(\mu_{f}\right)=\inf \left\{H D(Y): Y \subset X, \mu_{f}(Y)=1\right\}$. Note that $\kappa=h_{\mu_{f}}\left(T_{X}^{k}\right) / \chi$ (see [24], [19] or [20]).

Lemma 4.8. Let the assumptions of Theorem 4.3 be satisfied. Suppose that $\sigma^{2}$, defined in (4.3) for the function $g=\sum_{0 \leq j<k}\left(f-P(T, f)+\kappa \log \left|T^{\prime}\right|\right) \circ T^{j}$, is strictly positive. If $h$ belongs to the lower class (i.e. satisfies (4.4)), then for $\mu_{f}$ a.e. $x \in X$,

$$
\limsup _{U \downarrow x} \frac{m_{f}(U)}{d(U)^{\kappa} \exp \left(\sigma \chi^{-1 / 2} h(-\log d(U)) \sqrt{-\log d(U))}\right.}=0 .
$$

If $h$ is constant, then

$$
\limsup _{U \downarrow x} \frac{m_{f}(U)}{d(U)^{\kappa} \exp \left(\sigma \chi^{-1 / 2} h(-\log d(U)) \sqrt{-\log d(U))}\right.}=\infty .
$$


Moreover, if for $\mu_{f}$ a.e. $x \in X$, the set of all integers $n$, for which there exists an interval $U$ containing $x$ and satisfying $n(U)=n$ for some $n(U)$ defined by (4.7) and (4.8), has bounded gaps, then (4.14) continues to hold for all functions $h$ belonging to the upper class.

Remark. The sequence $n(U)$ has $\mu_{f}$ a.e. bounded gaps, if, for example, the system $\left(X, T_{X}\right)$ admits a Markov partition.

Proof. Use formulas (4.11) and (4.12) to show that for $x \in K$ and $U$ containing $x$,

$$
\begin{array}{r}
\left(C_{1} C_{3}\right)^{-1} \exp \left(S_{k n(U)}\left(f-P(T, f)+\kappa \log \left|T^{\prime}\right|\right)(x)\right. \\
\left.-\frac{\sigma}{\sqrt{\chi}} h(-\log d(U)) \sqrt{-\log d(U)}\right) \\
\leq \frac{m_{f}(U)}{d(U)^{\kappa} \exp \left(\sigma \chi^{-1 / 2} h(-\log d(U)) \sqrt{-\log d(U))}\right.} \\
\leq C_{1} C_{3} \exp \left(S_{k n(U)}\left(f-P(T, f)+\kappa \log \left|T^{\prime}\right|\right)(x)\right. \\
\left.-\frac{\sigma}{\sqrt{\chi}} h(-\log d(U)) \sqrt{-\log d(U)}\right) .
\end{array}
$$

By the ergodic theorem, the law of the iterated logarithm and (4.11),

$$
\mid \log d(U))+n(U) \chi \mid \leq 2 \sqrt{\sigma^{2}\left(\log \left|\left(T^{k}\right)^{\prime}\right|\right) n(U) \log \log n(U)} .
$$

If $h$ belongs to the lower class, Remark 4.4(4) shows that

$$
\underset{U \downarrow x}{\limsup \exp }\left(S_{k n(U)}\left(f-P(T, f)+\kappa \log \left|T^{\prime}\right|\right)(x)-\sigma h(\chi n(U)) \sqrt{n(U)}\right) \leq 1
$$

for $\mu_{f}$ a.e. $x$. Replacing $h$ by $h_{0}(t)=h(t / \chi)+a n^{-1 / 4}$ for some $a<0$ proves the lemma in case of lower class functions.

Finally, if $h$ is constant or belongs to the upper class, apply Lemma 4.7 or Remark 4.4(4) to obtain the other statements of the lemma in the same way.

The results about the relation between Hausdorff measures and equilibrium states are contained in the following two theorems. For a function $h:[1, \infty) \rightarrow$ $\mathbf{R}_{+}$define for sufficiently small $t>0$

$$
\tilde{h}(t)=t^{\kappa} \exp \left(\frac{\sigma}{\sqrt{\chi}} h(-\log t) \sqrt{-\log t}\right) .
$$

Theorem 4.9. Let the assumptions of Theorem 4.3 be satisfied, and suppose $\sigma^{2}>$ 0 , where $\sigma^{2}$ is defined by (4.3) for the function

$$
g=\sum_{0 \leq j<k}\left(f-P(T, f)+\kappa \log \left|T^{\prime}\right|\right) \circ T^{j} .
$$


Then

$$
\mu_{f} \ll H_{\tilde{h}} \text { if } h \text { belongs to the lower class }
$$

(cf. [15] for a definition of $H_{\tilde{h}}$ ) and

$$
\mu_{f} \perp H_{\hat{h}} \text { if } h \text { is constant. }
$$

Moreover, if for $\mu_{f}$ a.e. $x$, the sequence

$$
\{n \in \mathbf{N}: \exists U \text { such that } x \in U, n(U)=n\}
$$

has bounded gaps, then (4.16) holds for all functions $h$ belonging to the upper class.

Remark. Taking $h \equiv 0$ it follows from (4.16) that the measure $\mu_{f}$ is orthogonal to the $\kappa$-dimensional Hausdorff measure on $K$.

Proof. (1) Proof of (4.15): Let $h$ satisfy (4.4). By Lemma 4.8 (4.13), for a set $E$ with $\mu_{f}(E)>0$, there exist a set $E^{\prime} \subset E$ satisfying $\mu_{f}\left(E^{\prime}\right)>\mu_{f}(E) / 2$ and a $\theta>0$ such that for every $x \in E^{\prime}$ and all intervals $U$ of diameter less than $\theta$ containing $x, m_{f}(U)<\tilde{h}(d(U))$. Therefore $H_{\tilde{h}}(E) \geq H_{\tilde{h}}\left(E^{\prime}\right) \geq m_{f}\left(E^{\prime}\right)$. Since $\mu_{f} \ll m_{f}, m_{f}\left(E^{\prime}\right)>0$, whence $H_{\tilde{h}}(E)>0$ and $\mu_{f}$ is absolutely continuous with respect to $H_{\tilde{h}}$.

(2) Proof of (4.16): The additional statement concerning the formula (4.16) is shown exactly in the same way as it is proved for constant functions. It is only necessary to apply the corresponding statements from Lemma 4.8. Hence it suffices to show (4.16) for constant functions $h$.

For $n \geq 1$ and $\varepsilon>0$, by Lemma 4.8 (4.14), there exists a set $E_{n} \in \mathscr{F} \cap K$ such that $\mu_{f}\left(E_{n}\right)>1-\varepsilon 2^{-n}$ and such that for $x \in E_{n}$ and some suitable interval $U_{x}$ of diameter $<1 / n$ containing $x, m_{f}\left(U_{x}\right)>2 n \tilde{h}\left(d\left(U_{x}\right)\right)$. From the cover $\left(U_{x}: x \in E_{n}\right)$ of $E_{n}$ choose a subcover $\left(U_{l}: l \geq 1\right)$ of multiplicity $\leq 2$. Since $d(U)<1 / n$,

$$
H_{\tilde{h}}\left(E_{n}, \frac{1}{n}\right) \leq \frac{1}{2 n} \sum_{l=1}^{\infty} m_{f}\left(U_{l}\right) \leq \frac{1}{n} m_{f}(K) \leq \frac{1}{n} .
$$

Setting $F_{\varepsilon}=\bigcap_{n \geq 1} E_{n}$ it follows that $H_{\tilde{h}}\left(F_{\varepsilon}\right)=0$ and $\mu_{f}\left(F_{\varepsilon}\right) \geq 1-\varepsilon$. Finally the set $F=\bigcup_{l \geq 1} F_{1 / l}$ satisfies $H_{\hat{h}}(F)=0$ and $\mu_{f}(F)=1$, proving (4.16).

Theorem 4.10. Let the assumptions of Theorem 4.3 be satisfied, and suppose $\sigma^{2}=0$, where $\sigma^{2}$ is defined by (4.3) for the function

$$
g=\sum_{0 \leq j<k}\left(f-P(T, f)+\kappa \log \left|T^{\prime}\right|\right) \circ T^{j} .
$$

Then $H D\left(\mu_{f}\right)=H D(K)=\kappa$ and $\mu_{f}$ is equivalent to the $\kappa$-dimensional Hausdorff measure on $K=\operatorname{supp}\left(\mu_{f}\right)$.

Proof. Since $\sigma^{2}=0$ it is well known that $g$ is homologous to a constant function, and since $\int_{X} g d \mu_{f}=0$ this constant must be zero. In particular, 
$P\left(T_{X}^{k},-\kappa \log \left|\left(T^{k}\right)^{\prime}\right|\right)=0$. From this formula it follows that $H D\left(\mu_{f}\right)=\kappa($ see [10] and also [2], [14] or [19]).

The functions $\sum_{0 \leq j<k}(f-P(T, f)) \circ T^{j}$ and $\sum_{0 \leq j<k}\left(\kappa \log \left|T^{\prime}\right|\right) \circ T^{j}$ are homologous by a Hölder continuous function. Consequently, rewriting (4.12), there exists a constant $C>0$ such that for $x \in K$ and $r>0$

$$
C^{-1}\left|\left(T^{k n(x, r)}\right)^{\prime}(x)\right|^{-\kappa} \leq m_{f}\left(B_{X}(x, r)\right) \leq C\left|\left(T^{k n(x, r)}\right)^{\prime}(x)\right|^{-\kappa},
$$

where $n(x, r)=n(B(x, r))$. It is well known (and easy to show) that this formula, together with (4.11), imply that $m_{f}$ and $H_{t^{\kappa}}$ are equivalent on $K$ (even with bounded Radon-Nikodym derivatives). But $\mu_{f}$ and $m_{f}$ are equivalent on $K$, whence the theorem.

\section{REFERENCES}

1. R. Bowen, Equilibrium states and the ergodic theory of Anosov diffeomorphisms, Lecture Notes in Math., Vol. 470, Springer-Verlag, 1975.

2. $\ldots$, Hausdorff dimension of quasi circles, Inst. Hautes Études Sci. Publ. Math. 50 (1980), 11-25.

3. M. Denker, C. Grillenberger, and K. Sigmund, Ergodic theory on compact spaces, Lecture Notes in Math., Vol. 527, Springer-Verlag, 1976.

4. M. Denker, G. Keller, and M. Urbański, On the uniqueness of equilibrium states for piecewise monotone mappings, Studia Math. 97 (1990), 27-36.

5. K. J. Falconer, The geometry of fractal sets, Cambridge Univ. Press, New York, 1985.

6. F. Hofbauer and G. Keller, Ergodic properties of invariant measures for piecewise monotonic transformations, Math. Z. 180 (1982), 119-140.

7. __ Equilibrium states for piecewise monotone maps, Ergodic Theory Dynamical Systems 2 (1982), 23-43.

8. I. A. Ibragimov and Y. V. Linnik, Independent and stationary sequences of random variables, Wolters-Noordhoff, Groningen, 1971.

9. N. G. Makarov, On the distortion of boundary sets under conformal mappings, Proc. London Math. Soc. 51 (1985), 369-384.

10. H. McClusky and A. Manning, Hausdorff dimension for horseshoes, Ergodic Theory Dynamical Systems 3 (1983), 251-260.

11. M. Misiurewicz, $A$ short proof of the variational principle for $\mathbf{Z}_{+}^{N}$ action on compact space, Bull. Acad. Polon. Sci. Ser. Math. 24 (1976), 1069-1075.

12. S. J. Patterson, The limit set of a Fuchsian group, Acta Math. 136 (1976), 241-273.

13. __ Lectures on measures on limit sets of Kleinian groups, Analytic and Geometric Aspects of Hyperbolic Space (D. B. A. Epstein, ed.), LMS Lecture Notes Ser., Vol. 111, Cambridge Univ. Press, 1987.

14 F. Przytycki, M. Urbański, and A. Zdunik, Harmonic, Gibbs and Hausdorff measures on repellers for holomorphic maps. I, II, Ann. of Math. 130 (1989), 1-40 and Studia Math. 97 (1991), 189-225.

15 C. A. Rogers, Hausdorff measures, Cambridge Univ. Press, 1970.

16 D. Ruelle, Repellers for real analytic maps, Ergodic Theory Dynamical Systems 2 (1982), 99-107.

17 M. Shub and D. Sullivan, Expanding endomorphisms of the circle revisited, Ergodic Theory Dynamical Systems 5 (1985), 285-289.

18 D. Sullivan, Conformal dynamical systems, Geometric Dynamics, Lecture Notes in Math., Vol. 1007, Springer-Verlag, 1983, pp. 725-752. 
19. M. Urbański, Hausdorff dimension of invariant sets for expanding mappings of the circle, Ergodic Theory Dynamical Systems 6 (1986), 295-309.

20. _ Invariant subsets of expanding mappings of the circle, Ergodic Theory Dynamical Systems 7 (1987), 627-645.

21. _ Hausdorff dimension of invariant subsets for endomorphisms of the circle with an indifferent fixed point, J. London Math. Soc. 40 (1989), 158-170.

22. __ On Hausdorff dimension of the Julia set with a rationally indifferent periodic point, Studia Math. 97 (1991), 167-188.

23. P. Walters, Equilibrium states for $\beta$-transformations and related transformations, Math. $\mathrm{Z}$. 159 (1978), 65-88.

24. L. S. Young, Dimension, entropy and Lyapunov exponents, Ergodic Theory Dynamical Systems 2 (1982), 109-124.

INSTitut Für Mathematische Stochastik, Lotzestrasse 13, 3400 GötTingen, GermanY

Institute of Mathematics, Ul. Chopina 12, 87-100 ToRuń, Poland

Current address: Department of Mathematics, North Texas University, Denton, Texas 762035116 